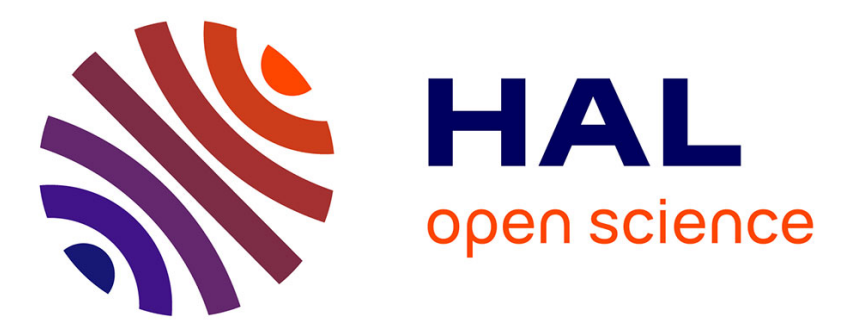

\title{
Tolerance analysis in manufacturing using the MMP Comparison and evaluation of three different approaches
}

\author{
Mojtaba Kamali Nejad, Frédéric Vignat, François Villeneuve
}

\section{To cite this version:}

Mojtaba Kamali Nejad, Frédéric Vignat, François Villeneuve. Tolerance analysis in manufacturing using the MMP Comparison and evaluation of three different approaches. 11th CIRP International Conference on Computer Aided Tolerancing, Mar 2009, Annecy, France. 10 p. hal-00366342v1

\section{HAL Id: hal-00366342 \\ https://hal.science/hal-00366342v1}

Submitted on 6 Mar 2009 (v1), last revised 14 Jan 2010 (v2)

HAL is a multi-disciplinary open access archive for the deposit and dissemination of scientific research documents, whether they are published or not. The documents may come from teaching and research institutions in France or abroad, or from public or private research centers.
L'archive ouverte pluridisciplinaire HAL, est destinée au dépôt et à la diffusion de documents scientifiques de niveau recherche, publiés ou non, émanant des établissements d'enseignement et de recherche français ou étrangers, des laboratoires publics ou privés. 


\title{
Tolerance analysis in manufacturing using the MMP Comparison and evaluation of three different approaches
}

\author{
Mojtaba Kamali Nejad, Frédéric Vignat, François Villeneuve \\ University of Grenoble, G-SCOP Laboratory \\ 46, avenue Félix Viallet - 38031 Grenoble Cedex 1 - France \\ Mojtaba.Kamalinejad@g-scop.inpg.fr
}

\begin{abstract}
In previous works, the authors have developed the Model of Manufactured Part (MMP) [1], a method for modeling the different geometrical deviation impacts on the part produced (error stack-up) in a multi-stage machining process. They also proposed different solution techniques to identify the worst case for the purpose of tolerance analysis. The first proposed solution technique consists of optimization techniques as Sequential Quadratic Programming (SQP) or Genetic Algorithm (GA) [2]. The second one combines the MMP and the Jacobian-Torsor Model [3] [4] that benefits of the interval arithmetic advantages to solve the worst case searching. The last technique uses Monte Carlo simulation to generate a population of virtually manufactured parts representative of the real produced parts [5]. This paper first reminds the MMP model and the different solution techniques. The different strategies to simulate the deviation parameters of the model are then discussed. For each of the three proposed solution techniques, its convenience and inconvenience is explored in detail. The solution techniques performances are compared from different points of view (i.e. rapidity, convergence to the global minimum, analyzed case ...) and some perspectives are presented.
\end{abstract}

\section{Keywords:}

Tolerance analysis, Process plan, Errors stack up, Model of Manufactured Part

\section{INTRODUCTION}

Today, manufacturing engineers are faced with the problem of selecting the appropriate process plan (machining processes and production equipment) to ensure that design specifications are satisfied. Developing a suitable process plan for release to production is complicated and time-consuming. Currently, trial runs or very simple simulation models (1D tolerance charts for example [6]) are used to check the quality criterion. The trial runs are very costly and, on the other hand, the accuracy of simulation fails to meet today's requirements. These problems can be overstepped by developing accurate models and methodologies for simulating the manufacturing process and predicting geometrical variations in the parts produced. More accurate models will make it possible to evaluate the process plan, determine the tolerance values in terms of manufacturing capabilities during the design phase, and define the manufacturing tolerances to be checked for each setup. In the literature available on this subject, the evaluation of a process plan in terms of functional tolerances is called the tolerance analysis. In this paper the model of manufactured part (MMP) is used for simulating the manufacturing process and then the worst case technique and statistical approach are used for the aim of tolerance analysis.

In this paper we shall focus on tolerance analysis relating to error propagation in a multi-stage machining process. Huang et al [7] propose a state space model to describe part error propagation in successive machining operations. Surface deviation is expressed in terms of deviation from nominal orientation, location and dimensions. The error sources in machining operations are classified as fixture errors, datum errors and machine tool errors. A part's deviation is expressed in terms of the deviation of its surfaces and is stored in a state vector $x(k)$. This vector is then modified by moving from

operation $k$ to $k+1$. Zhou et al [8] uses the same state model but the surface deviation compared with the nominal state is expressed using a differential motion vector. However, these two models require specific fixture setups (e.g., an orthogonal 3-2-1 fixture layout). More recently, Loose et al [9] used the same state space model with a differential motion vector but including general fixture layouts. Although a general fixture layout is considered, the error calculation of a fixture is based on its locator deviations (a locator is a punctual connection). Hence, positioning cases with Plane/Plane contact or Cylinder/Cylinder floating contact are not envisaged.

Huang et al [10] propose a simulation-based tolerance stack-up analysis. Manufacturing errors are classified as follows: work holding errors (i.e. fixture errors, datum errors and raw part errors), machine tool errors and cutting tool errors. A surface is modeled using uniformly distributed sample points (point cloud), which is a basic technique applied in CMM type inspections. By putting the part through different machining setups, the coordinates of these points in the local part coordinate system are changed due to manufacturing errors. The Monte Carlo method is used to perform the simulation. The different possible errors are considered in this simulation but Part/Fixture interaction is not studied and it is assumed that part/fixture contact is perfect.

This paper firstly reminds the Model of Manufactured Part (the MMP) $[1,11,12]$; a method for modeling the different geometrical deviation impacts on the part produced (error stack-up) in a multi-stage machining process. Previously, the same authors presented a generic formulation for tolerance analysis based on searching for the worst case using the MMP. This paper discusses about the different numerical solution technique for performing the worst case based tolerance analysis. Worst case technique then will be compared with statistical tolerance analysis. The statistical approach uses the Monte Carlo Simulation. The convenience and inconvenience of each technique will be then discussed.

\section{MMP}

$[1,11,12]$ propose a method for modeling successive machining processes that takes into account the geometrical and dimensional deviations produced with each machining setup and the influence of these deviations on further setups. In the MMP, the errors generated by a manufacturing process are considered to be the result of two independent phenomena: Positioning 
and Machining. These deviations are accumulated over the successive setups (See Fig. 1). The result is expressed in terms of deviation of the actual surfaces compared with those of the nominal part. In order to capture the error stacks, an intermediate virtual part (MWP) is put through the different setups. In setup $k$, the machined surface deviation is the combination of positioning errors and machining errors. Positioning errors are caused by surface deviations from a previous setup (datum errors) and fixture surface deviations in setup $\mathrm{k}$. Machining errors are machined surface deviations compared with the nominal position in the machine tool in setup $k$. These errors stem from multiple and various sources ranging from machine geometry and control to cutting deformations.

At the end of the modeling process, a virtual manufactured part (MMP) is created. This MMP stores data about the deviations generated (combination of parameters and range of variation) during the full machining process. See Fig. 1.

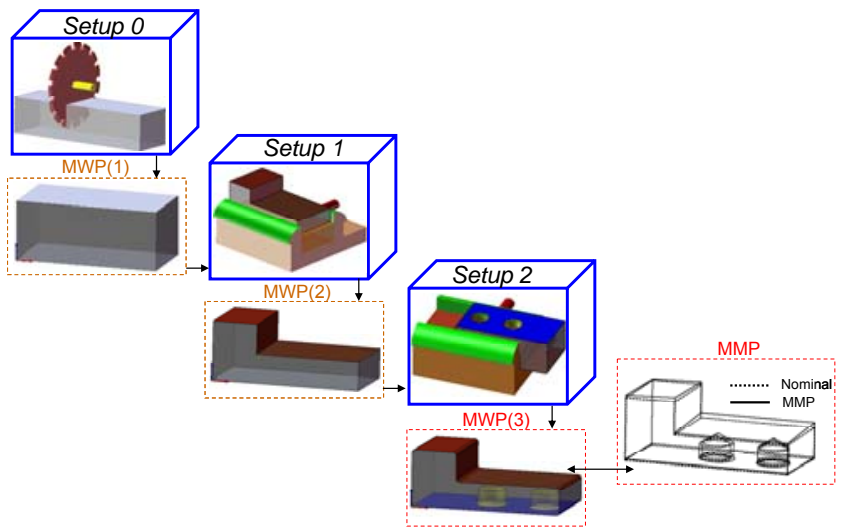

Figure 1 : Tolerance stack-up model

The MMP does not only represent a model of one manufactured part containing a description of the process in terms of geometrical deviations and accumulated defects. In fact, because it indicates the variation range of the generated defects it represents the series of parts produced. The model describes the defects, classifies them and indicates their variation range.

Small Displacement Torsors (SDT) describes the MMP surface deviations, i.e. the MMP parameters, which can be classified according to four categories.

- $\quad$ Machining deviations -DM- $\left(\left(r x_{i}, r y_{i}, t z_{i} \ldots\right)\right.$

- $\quad$ Fixture surface deviations $-\mathrm{DH}-\left(r x_{i S j}, t x_{i S j}, \ldots\right)$

- $\quad$ Link parameters -LHP-( $\left.\quad \operatorname{lr}_{i S j}, \operatorname{lt}_{i G j} \ldots\right)$

- $\quad$ Actual surface deviations relative to the nominal part $\left(r x_{P, P i}, r y_{P, P i}, \ldots\right)$

The machining deviation parameters (DM) are limited by constraints (CM) representing machine and tools capabilities. The $\mathrm{DH}$ parameters are limited by constraints $(\mathrm{CH})$ representing the fixture quality.

Due to the type of connection (floating or slipping), the link parameter values (LHP) are determined by a specific algorithm (CHP) including constraints and, in certain cases, a positioning function.

For each MWP surface made, the positioning and machining deviations are added. The deviation relative to the nominal part is determined and expressed as $T_{P, P i}$ for surface $i$ of the MWP. See (1). This Torsor will be kept in the MWP data for possible further use in another setup for an assembling procedure or for the purposes of tolerance analysis.
For the example of Figure 1, surface deviations relative to the nominal part regarding surface 3 is expressed as Equation (1).

$T_{P, P 3}=\left[\begin{array}{cc}r x_{P, P 3} & 0 \\ r y_{P, P 3} & 0 \\ 0 & t z_{P, P 3}\end{array}\right]_{L C S 3} \quad$ Where : $\left\{\begin{array}{l}\mathrm{tz}_{\mathrm{p}, \mathrm{P3}}=7.07 \mathrm{lrx}_{1 \mathrm{S2}}+0.7\left(-\mathrm{ltz}_{1 \mathrm{S2}}+\mathrm{ltz}_{2 \mathrm{~S} 2}\right)-7.07 \\ \mathrm{rx}_{1}+7.07 \mathrm{rx}_{152}+0.7\left(-\mathrm{tz}_{1}-\mathrm{tz}_{152}+\mathrm{tz}_{2}+\mathrm{tz}_{2 \mathrm{SS}}\right)+\mathrm{tz}_{3}\end{array}\right.$

\subsection{Tolerance Analysis and Virtual Gauge}

Two techniques have been used in tolerance analysis: worst case analyzing and statistical analyzing. In worst case tolerance analyzing (WC), it is assumed that all deviations could simultaneously occur at their worst limits. The extreme value of a functional tolerance under any possible variations caused by deviations is determined. In statistical tolerance analysis, it is assumed that individual deviations are independent and have some frequency distribution, which allows computing the probability that the product can be found at the end of machining and will function under given individual tolerances, often by Monte-Carlo methods.

In this paper, both of the above mentioned tolerance analysis techniques will be applied with the MMP. First of all, we introduce how we check the conformity of an individual part with the functional tolerances by means of a virtual gauge. Secondly the worst case searching in section 3 and statistical tolerance analysis in section 4 will be followed. Because of the different nature of WC tolerance analysis and statistical analysis, they will be presented in two separate sections.

In the present work, functional tolerance compliance is checked using a virtual gauge. Each tolerance is modeled by a virtual gauge according to the standard concerned. A virtual gauge is a nominal part made up of positioning surfaces (in red) and tolerance zones (in green). This gauge is assembled with the MMP according to the chosen standard rules (usually ISO or ASME). The Gauge and MMP assembling process is based on a set of hierarchically organized elementary connections. The Gauge/MMP assembly link parameter values (LGP) are determined by a specific algorithm (CGP) similar to the CHP algorithm used to calculate the MWP/Fixture assembly link parameters. Once the gauge and MMP assembly is finished, functional tolerance compliance is verified by the GapGP $_{k}$ signed distance measured between the virtual gauge modeling the tolerance and the MMP surfaces concerned. This distance is measured at the necessary points along the boundary of the toleranced surface. The distance with the positive sign represents a point inside the tolerance zone and a distance with a negative sign represents a point outside of tolerance zone.

\section{WORST CASE SEARCHING}

If the worst part in a series of manufactured parts complies with the functional tolerance, it is logical to conclude that all of the parts manufactured will comply. This technique is a little far from reality. It means that even if the worst part in series of manufactured parts does not comply with the functional tolerance, the much percent of manufactured parts could be acceptable. How ever this technique is largely used in the manufacturing of high precision parts like for the plane engines and army. We developed two techniques for worst case searching associated with MMP: optimization technique and combined approach. 


\subsection{Optimization technique}

A generic formulation of the worst case searching problem, as proposed by Villeneuve et al [1], consists in solving the following objective functions:

$$
\left.\min _{D M, D H, L H P}^{C M, C H, C H P}\left(\max _{L G P}^{C G P}\left(\operatorname{GapGP}_{\min }\right)\right)\right)
$$

These functions express the search for the worst case in terms of the functional tolerance under analysis. A process plan is considered able to satisfy the functional tolerance if the value determined in Equation. (2) remains positive or null while the MMP parameters vary in their limited variation domain. As previously underlined, functional tolerance compliance is verified by the signed distance $\mathrm{GapGP}_{\mathrm{k}}$. For a given problem, the critical distance is the minimum distance denoted by GapGP $_{\text {min }}$ as developed in Eq. (3).

$$
\operatorname{Gap} G p_{\min }=\min _{k=1,2, \ldots}\left(G a p G P_{k}\right)
$$

The Gauge/MMP assembly is not always complete and some limited relative displacements remain possible due to the material condition modifiers, incomplete datum frames or the type of tolerance (i.e. orientation tolerance). These displacements may correspond to the degrees of freedom ( $\left(\mathrm{GP}_{\mathrm{DOF}}\right)$ of the Gauge/MMP assembly or to the parameters of a floating contact $\left(\mathrm{LGP}_{\mathrm{f}}\right)$. In the second case, the displacements are described by the link parameters and their limits by the positioning algorithm CGP (generally non-penetration condition) complying with the chosen tolerancing standard (ISO, ASME). Within the limits of these displacements, the most favorable position for the virtual gauge relative to the MMP can thus be found. In this position, the GapGP ${ }_{\text {min }}$ has a maximum value. In other words, the virtual gauge will be displaced by an optimization algorithm that explores possible displacements until the best position with respect to the MMP is found. In this position, all the $\mathrm{GapGP}_{k}$ will be measured for functional tolerance verification. This procedure is expressed by Eq. (4).

$$
\underset{L G P}{C G P}(\ldots))
$$

The GapGP* solution provided by Eq. (4) is also interpreted as a virtual measurement of an individual part. As previously stated, the method presented in this study for analyzing functional tolerance consists in finding the worst case (minimum value of the GapGP*) The search for the worst case is an optimization task that can be expressed as shown below, Eq. (5). The objective function in this optimization is the GapGP*. The variables are the $\mathrm{CM}, \mathrm{CH}$, and LHP. The limits of these variables are expressed by constraints $(\mathrm{CM}$ and $\mathrm{CH})$ and the positioning algorithm (CHP).

$$
\min _{D M, D H, L H P}^{C M, C H, C H P}(\ldots)
$$

\section{Technique used in the solution}

Eq. (2) is a multi-layer constrained optimization problem. It checks whether a process plan is able to satisfy functional tolerance requirements. In order to provide a clearer mathematical representation of Eq. (2), a new formulation for worst case identification shall be put forward in this section. Secondly, a technique for solving the worst case search issue shall be discussed.
To simplify the technique adopted, the problem is broken down into two sub problems, Eqs. (6) and (7), and variable substitution is applied. [2]

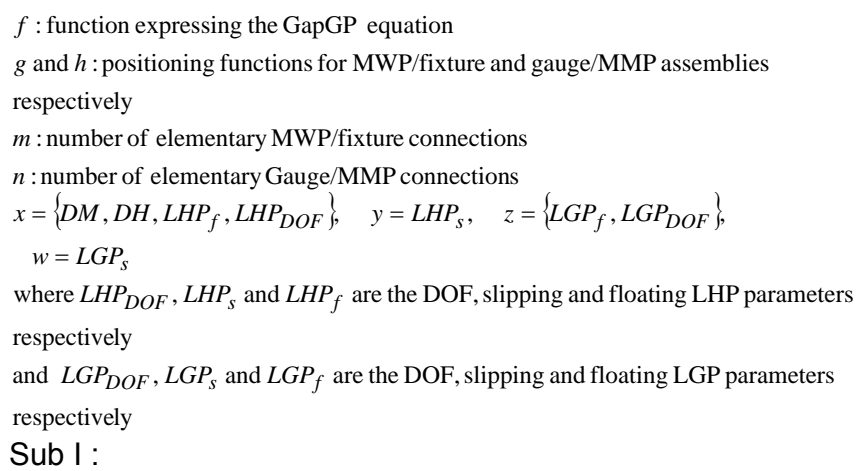

Worstcase value $=\min F(x, y)$

$$
\begin{aligned}
& \text { Subject to: } c(x, y) \geq 0 \\
& \text { Where: } y=\left\{y_{1}, y_{2}, \ldots, y_{i}\right\} \\
& \text { With: } \forall i \in[1, m], y_{i}=\text { Solution of }: \max _{y_{i}} g_{i}\left(y_{i}\right)
\end{aligned}
$$

Sub II : Subject to: $c(x, y) \geq 0$

$$
\begin{aligned}
& F(x, y)=\operatorname{maxmin}_{z}\left\{f_{i}\right\} \\
& \quad \text { Subject ta } c(x, y, z, z, w) \geq 0 \\
& \text { Where: } w=\left\{w_{1}, w_{2}, \ldots, w_{i}\right\} \\
& \text { With: } \forall i \in[1, n], w_{i}=\text { Solutionof }: \max _{w_{i}} h_{i}\left(w_{i}\right) \\
& \text { Subject ta } c(x, y, z, w) \geq 0
\end{aligned}
$$

The task in Sub $\mathrm{I}$ is to find the worst possible part produced in a multi-stage machining process in relation to the tolerance being analyzed. The task in Sub II is to perform a virtual measurement of one individual part. In Sub II, the value of $F(x, y)$ is calculated and supplied to Sub I. To be able to solve the positioning algorithms (CHP and CGP), each sub problem is broken down into different layers. The readers are addressed to [2] for more information and the details of Sub problems and the layers concerned.

\section{Solution strategy}

Based on the constraints associated with $\mathrm{DM}$ and $\mathrm{DH}$, the proper optimization algorithm should be applied. One iteration in level of Sub I follows with many iterations in Sub II and its layers. If the gradient optimization method is used for Sub I, the initial gauss plays a very important role. If the wrong gauss is chosen, there is a high risk of reaching a local minimum (instead of a global minimum).

Stochastic methods such as genetic algorithms can be applied as well. The advantage of this kind of algorithm is that, by creating a big enough population, the probability of finding the global minimum point is higher. The drawback is the time needed to run such an algorithm.

\section{Quality constraints}

The machined surface deviation torsor parameters, known as machining deviation parameters (DM), are limited by constraints (CM) stemming from the machine tool capabilities. The parameters of the fixture surface deviation torsor $(\mathrm{DH})$ are also limited by constraints $(\mathrm{CH})$ arising from the fixture quality. These constraints limit either one or a set of parameters. There are 3 main strategies for defining these constraints.

\section{$1 \quad$ Using the Measurement Results}

In this strategy, a sufficient number of parts have to be produced and measured. The manufacturing conditions (temperature, machine tool, etc.) should be the same as 
for the simulation. The machined surface or positioning surface deviation ranges are obtained from the measurement data. Readers can refer to [13] for more details about how the measurements are performed. In this strategy, the measurement results will be modeled, assuming that the deviation parameters are not independent. Based on the measurement results obtained, the co-relation between the parameters is then sought (See Fig. 6). This strategy is very close to reality, but it is complicated to express the co-relation between the parameters.

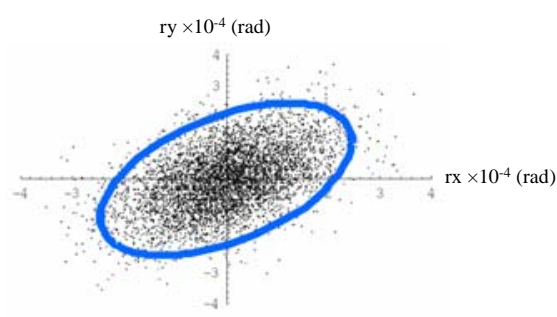

Figure 2 : Co-relation between deviations parameters ( $r x$, ry) [14]

With this strategy, the constraints obtained for the deviation parameters can be expressed as Eq. (8).

$a \cdot r x^{2}+b \cdot r y^{2}+c \cdot r x \cdot r y \leq d$

.2

Using the Measurement Results (independent parameters)

As explained for the previous strategy, the parameter deviation ranges are obtained by measurements. As opposed to the previous strategy, the parameters here are assumed to be independent variables (for the purposes of simplification) with a normal distribution that varies in the interval defined by $[-3 \sigma+3 \sigma]$. With this strategy, the constraints obtained will be as in Eq. (9) in the case of a plane SDT. The deviation variation range obtained is close to reality but considering independent parameters implies that these they can simultaneously attain their extreme limits. This is highly improbable in reality.

$$
\begin{aligned}
& \underline{r x} \leq r x \leq \overline{r x} \\
& r \underline{r y} \leq r y \leq \overline{r y} \\
& \underline{t z} \leq t z \leq \overline{t z}
\end{aligned}
$$

$$
.3
$$

\section{Considering a Variation zone with dependent} parameters

In this strategy, a standard variation zone is used to represent the deviation range of a surface or its feature (axis, center, etc.). Desrochers [4] proposes a 3-D representation of the variation zones. A variation zone can be used in its generic form to present the potential variations along and about all the three Cartesian axes. The proposed representation comprises all standard variation zones, along with their corresponding SDT representation and geometrical constraints. The SDT parameter variations must be bound by the limits of the 3$D$ variation zones they represent. These boundary areas are hyper-surfaces of the space spanned by the six small displacement variables ( $r x, r y, r z, t x, t y, t z$ ). Illustrated below is the case of a planar variation zone showing how such constraints can be handled. In Figure 3, the variation zone is defined as the volume ranging between two parallel planes with a distance $e$ between them. The ideal associated plane (shaded in Figure 3 must therefore lie inside this zone. The boundary points will be used to ensure that the associated surface remains within the variation zone. If four boundary points $(A, B, C$ and $D)$ are used on the associated plane with reference point $O$ at the barycenter, it is possible to express their projection on the limiting planes, yielding to the linear set of inequalities in Eq.10), where a, b and $e$ are known.

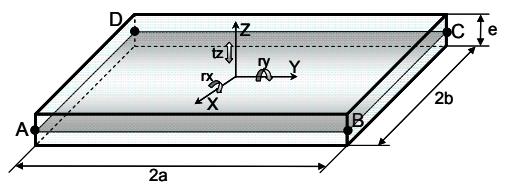

Figure 3 : Planar Variation Zone

$\left[\begin{array}{c}\frac{-e}{2} \\ \frac{-e}{2} \\ \frac{-e}{2} \\ \frac{-e}{2}\end{array}\right] \leq\left[\begin{array}{ccc}-a & b & 1 \\ -a & -b & 1 \\ a & -b & 1 \\ a & b & 1\end{array}\right] \bullet\left[\begin{array}{c}r x \\ r y \\ t z\end{array}\right] \leq\left[\begin{array}{c}\frac{e}{2} \\ \frac{e}{2} \\ \frac{e}{2} \\ \frac{e}{2}\end{array}\right]$

\subsection{Combined approach}

The second approach for worst case searching is combined approach. We built this combined approach on two existing models (the MMP and the Jacobian-Torsor model) for tolerance analysis in successive machining operations. It should be mentioned that the notation of the MMP and Jacobian-Torsor model has been homogenized in this paper for better understanding. This approach combined the benefits of the Jacobian and torsor approaches developed for tolerancing. The proposed approach is formulated using interval-based arithmetic.[4]

This section presents the combined approach and a worst case based analysis of the functional tolerances. Firstly the functional elements will be introduced and then the formulation will be presented.

\section{THE COMBINED APPROACH FUNCTIONAL ELEMENTS (FE)}

Basically we consider three types of uncertainties as functional elements (FE) deviations: machined surface deviation, fixture surface deviation and link. In other word every active surface which takes part in machining operation and the links are the functional elements. The possible deviations concerning the functional elements are expressed by small displacement torsors (SDT) with interval.

\section{Surface deviation representation by SDT with interval}

The SDT of a functional element reflects the deviation of the associated surface compared with its nominal position and is expressed in the local coordinate system of the FE concerned. This torsor will be used to express the machined surface deviations (machining errors) and the fixture surface deviations. The SDT can be represented by intervals where $(\underline{r x}, \underline{r y}, \underline{r z}, \underline{t x}, \underline{t y}, \underline{t z})$ and $(\overline{r x}, \overline{r y}, \overline{r z}, \overline{t x}, \overline{t y}, \overline{t z})$ signify the lower and upper limits of the small displacements rx, ry, rz, tx, ty and tz accordingly.

Equation (11) shows the SDT with intervals for the $i^{\text {th }}$ functional element:

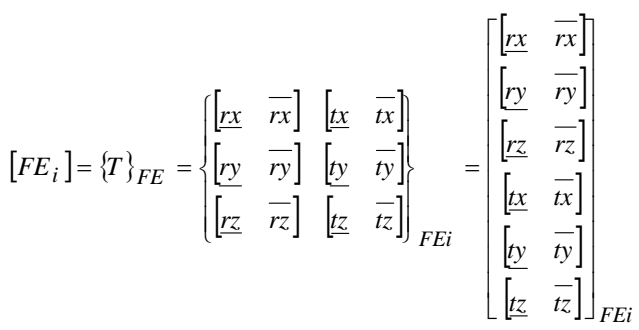




\section{Link representation by SDT with interval}

The scope of variation of the link parameters can be expressed using intervals. To obtain the variation interval of the link parameters, it is necessary to identify the different possible contacts for a link. Here, we have used the method proposed by Villeneuve et al [12]. This expresses the variation interval of the link parameters using an optimization problem. If the link parameters are considered as independent variables, their extreme bounds can be defined as shown in equation (12 and 13)

Case of MWP/Fixture:

$$
\overline{l *}=\underset{D H, D M, L H P}{\operatorname{Max}}(l *), \underline{l}=\underset{D H, D M, L H P}{\operatorname{Min}}(l *)
$$

Where $l *$ is the considered link parameter and $* \in\left\{r x_{k S j}, r y_{k S j}, r z_{k S j}, t x_{k S j}, t x_{k S j}, t x_{k S j}\right\}$

\section{Case of Gauge/MMP}

$$
\overline{l *}=\underset{D M, L G P}{\operatorname{Max}}(l *), \underline{l *}=\underset{D M, L G P}{\operatorname{Min}}(l *)
$$

Where $l *$ is the considered link parameter and $* \in\left\{r x_{k G j}, r y_{k G j}, r z_{k G j}, t x_{k g j}, t x_{k G j}, t x_{k G j}\right\}$

$$
\mathrm{k}=\text { surface number and } \mathrm{j}=\text { setup number }
$$

The link lower limit and upper limit values obtained will then be replaced in the link torsor as shown in equation (14).

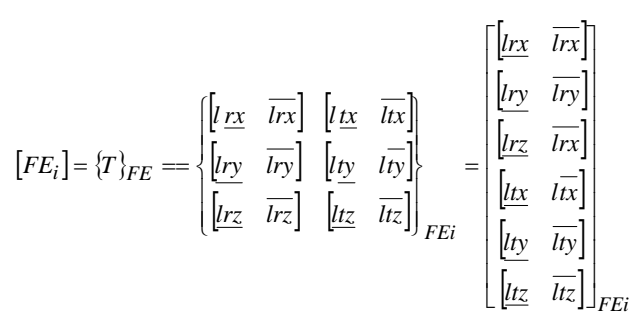

As previously underlined, according to the type of connection (floating or slipping), the link parameter values (LHP) are determined by a specific algorithm (CHP) that takes into account the constraints and, in certain cases, a positioning function.

\section{Tolerance analysis with the combined approach}

By applying some modifications to the Jacobian -Torsor model and combine it with the MMP a new method for tolerance analysis of manufactured parts has been developed. The defects are accumulated on the virtual manufactured part (MMP) and the compliance of the MMP and the functional tolerance can be checked by the GapGP $_{k}$ that can be calculated by equation (15).

$$
[\mathrm{GapGP}]=[\mathrm{A}][\mathrm{FEs}]
$$

Where:

[GapGP]: signed distance between virtual gauge and MMP, measured relative to the boundary of the tolerance zone

[FEs]: Functional Elements SDT or link torsor

$[\mathrm{A}]$ : Coefficient matrix expressing the geometrical relation between [GapGP] and [FEs]

Equation (15) is obtained from the virtual assembly of the MMP and the virtual gauge. Matrix $A$ expresses the contribution of FE deviation into the GapGP distances. Concerning the column of FE in the right hand side of equation (15), the machined surfaces deviation (machining errors) and fixture surfaces deviation can be obtained for the specific machines and fixtures and should be kept in a data base for using in combined approach. The variation interval of link elements could be calculated by equation (12 and 13).

It is possible to check the compliance of the parts to the functional tolerances with the sign of the extreme values of the $\mathrm{GapGP}_{\mathrm{k}}$ intervals. The process compliance condition for manufacturing good parts can be expressed as:

For $\mathrm{i}=1$ to number of verification point

$$
\sup \left\{G a p G P_{i}\right\} \geq 0 \text { And } \inf \left\{G a p G P_{i}\right\} \geq 0
$$

More details and information concerning the combined approach is available in [4].

\section{MONTE CARLO (MC) SIMULATION}

The method associated with MC consists of producing an enough number of parts to check whether they all comply with the functional tolerance. The parts are virtually produced according to the defects generation procedure. The defects generation procedure should be compatible with the chosen quality constraints.

Actually in Sub I, the DM, DH and $\mathrm{LHP}_{\mathrm{f}}$ parameters, which are represented by $\mathrm{x}$, are generated randomly within the domain limited by the $\mathrm{CM}, \mathrm{CH}$ and $\mathrm{CHP}$. The random generation of the parameters is established in such a way that their distribution be uniform on the defined variation scope.

As we have seen in 3 , there are different possibilities for defining the constraints associated with $\mathrm{DM}$ and $\mathrm{DH}$. Based on the chosen type of constraints, the DM and DH parameters should be generated for MC. For example if we consider the variation zone with dependent parameters, the DM and DH parameters cannot be generated directly using a random generator. Two different strategies are thus applied depending on the type of variation zone. For the cylindrical zone, a variable substitution is applied to be able to generate the 4 variables with independent variances according to (16). The 4 defect parameters describing the cylinder "real" position are then calculated using (17). [5]

$$
\begin{aligned}
& \mathrm{r}_{\mathrm{h}}=\left\{x \mid \begin{array}{l}
0 \leq \mathrm{x} \leq r_{\text {variation zone }}, \\
\text { probabilit y density } \mathrm{F}_{\mathrm{r}}(\mathrm{x})=\left(\frac{x}{r_{\text {variation zone }}}\right)^{2}
\end{array}\right\} \\
& \mathrm{r}_{\mathrm{l}}=\left\{x \mid \begin{array}{l}
0 \leq \mathrm{x} \leq r_{\text {variation zone }} \\
\text { probabilit y density } \mathrm{F}_{\mathrm{r}}(\mathrm{x})=\left(\frac{x}{r_{\text {variation zone }}}\right)^{2}
\end{array}\right\} \\
& \theta_{\mathrm{h}}=\left\{x \mid 0 \leq \mathrm{x} \leq 2 \pi \text { with probabilit y density } \mathrm{F}_{\theta}(\mathrm{x})=\left(\frac{x}{2 \pi}\right)\right\} \\
& \theta_{\mathrm{l}}=\left\{x \mid 0 \leq \mathrm{x} \leq 2 \pi \text { with probabilit y density } \mathrm{F}_{\theta}(\mathrm{x})=\left(\frac{x}{2 \pi}\right)\right\}
\end{aligned}
$$

$r x=-r_{h} \sin \left(\theta_{h}\right)+r_{l} \sin \left(\theta_{l}\right)$

$r y=r_{h} \cos \left(\theta_{h}\right)-r_{l} \cos \left(\theta_{l}\right)$

$t x=\frac{r_{h} \cos \left(\theta_{h}\right)+r_{l} \cos \left(\theta_{l}\right)}{2}$

$t x=\frac{r_{h} \sin \left(\theta_{h}\right)+r_{l} \sin \left(\theta_{l}\right)}{2}$ 

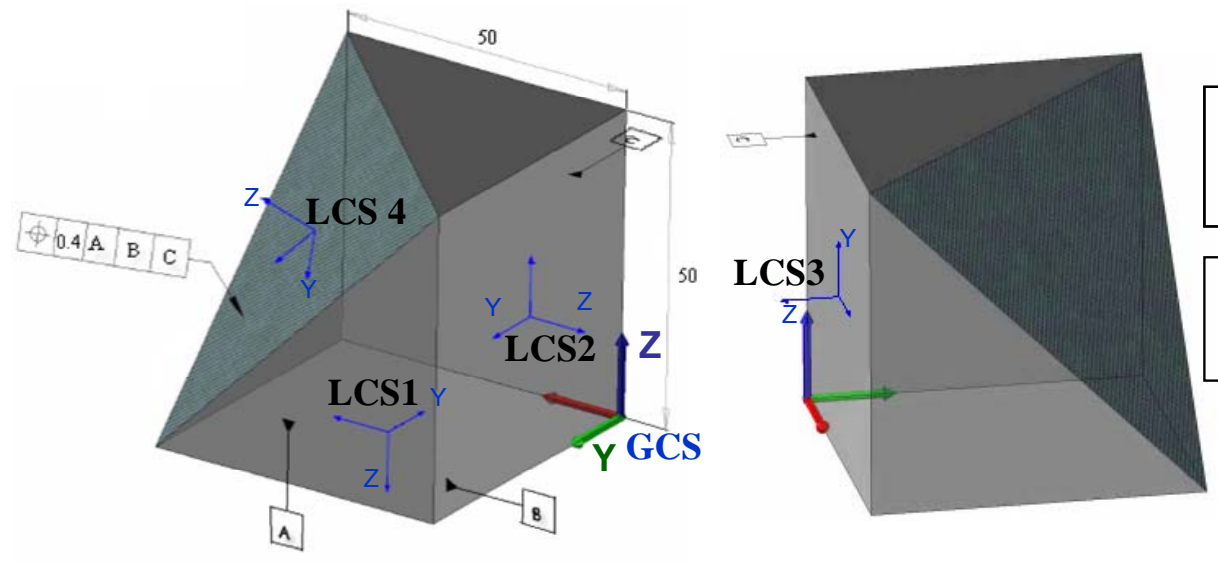

Setup1:

Raw part preparation by a sawing operation

\section{Setup 2:}

Positioning by: surface 1,2 \& 3 Milling surface 4

${ }^{*}$ GCS: Global Coordinate system

*LCS: Local Coordinate system

Figure 4 : Definition of 3D example

For the planar variation zone as illustrated in Figure 3 each nominal vertex $(A, B, C$ or $D)$ of the nominal plane is randomly displaced to create a "real" vertex. The direction of the displacement is normal to the nominal plane and the value of the displacement is randomly generated between $-\mathrm{e} / 2$ and $\mathrm{e} / 2$ with a uniform density. In other hand 4 random value between $-\mathrm{e} / 2$ and $\mathrm{e} / 2$ will be generated (called $X_{1}, X_{2}, X_{3}$ and $X_{4}$ ). A "real" plane is then positioned from the "real" vertices using a mean square root criteria. The 3 defect parameters $\mathrm{rx}$, ry and tz are then calculated from the "real" plane characteristics

For determining the link parameters (LHP) in a same way it should be considered hat they have to respect the positioning algorithm (CHP). The second requirement, for the determination of the link values, is that their density has to be uniform in the variation scope allowed by CHP.

More information regarding MC associated with the MMP is available in [5].

\section{EXAMPLE AND COMPARISON}

In this section, the three before mentioned techniques will be applied to a $3 \mathrm{D}$ example and their characteristics will be explored.

In the first section the quality constraints with independent parameters will be studied and in second section the quality constraints with dependent parameters will be discussed. Actually, quality constraints with independent parameters can be treated by all before mentioned techniques, but the quality constraints with dependent parameters can only be treated by optimization technique and MC. The part illustrated in Figure 4 will be used to perform a tolerance analysis of the double inclined machined plane with the before mentioned techniques.

Setup 1 of the process plan consists in preparing the raw blocks through a sawing operation. This results in the MWP that will go through setup 2. In setup 2, plane 4 is machined on a milling machine.

\subsection{First section}

\section{Combined approach}

The approach is nonetheless limited by the fact that it considers the parameters independently. In other word, today we can not consider the dependent DM and $\mathrm{DH}$ parameters with combined approach.

So in this section the quality constraints "Using the Measurement Results (independent parameters)" are considered and the MWP positioning surface deviation range, the machining errors and the fixture surface deviation range are obtained from a database created over the measurement results (the ranges are available in
Table 5 ). This section will not describe how to create the database (details about database creation can be found in [13]).

In the MWP/Fixture assembly in setup 2, the positioning surfaces of the MWP are plane 1, plane 2 and plane 3 , and the positioning surfaces of the fixture are plane 1S2, plane $2 \mathrm{~S} 2$ and plane $3 \mathrm{~S} 2$. The assembling procedure for the MWP/Fixture assembly comprises three hierarchical slipping connections. For each elementary connection, the link parameters (LHP) and positioning algorithm (CHP) are given in Table 2. Then, the MMP is obtained by simulation of the manufacturing process.

A virtual gauge is then created based on the functional tolerance. The gauge and the MMP are assembled to check that the functional tolerance is satisfied. The links between the MMP and the Gauge are formed of three hierarchical slipping connections. The MMP positioning surfaces are plane 1 , plane 2 and plane 3 and the related surfaces of the Gauge are plane 1G1, plane 2G1 and plane $3 \mathrm{G} 1$. The assembling process is similar to that of the MWP/fixture process so further details shall not be given here. The machined plane 4 is measured by means of 3 verification points that lead to 6 GapGP distances. The final objective in this step is to find the coefficient matrices $[A]$ (the obtained coefficient matrices are given in Table 5). In this step the extreme bounds of the link parameters related to the MWP/Fixture and Gauge/MMP assembly are calculated using equation (12 and 13). In the last step, the $6 \mathrm{GapGP}_{\mathrm{k}}$ variation intervals are calculated using Equation (15). See Table 3.

\section{Optimization Techniques}

The quality constraints for defining the MWP positioning surface deviation range, the machining errors and the fixture surface deviation range in setup 2, are already presented (Table 5) by "Using the Measurement Results (independent parameters)"The assembling process of MWP/fixture and those of Gauge/MMP are explained just before.

To be able to validate the process plan for satisfying the localization tolerance of plane 4 , the worst case is sought. In Sub II, there is no degree of freedom or floating link for the Gauge/MMP assembly.

The MATLAB software was used for programming with a Pentium $囚, 3.2 \mathrm{GHz}$. Both optimization algorithms (GA and SQP) were applied to minimize Sub I. Figure 6 represents the results obtained using the GA and Figure 5 represents those obtained using SQP. 

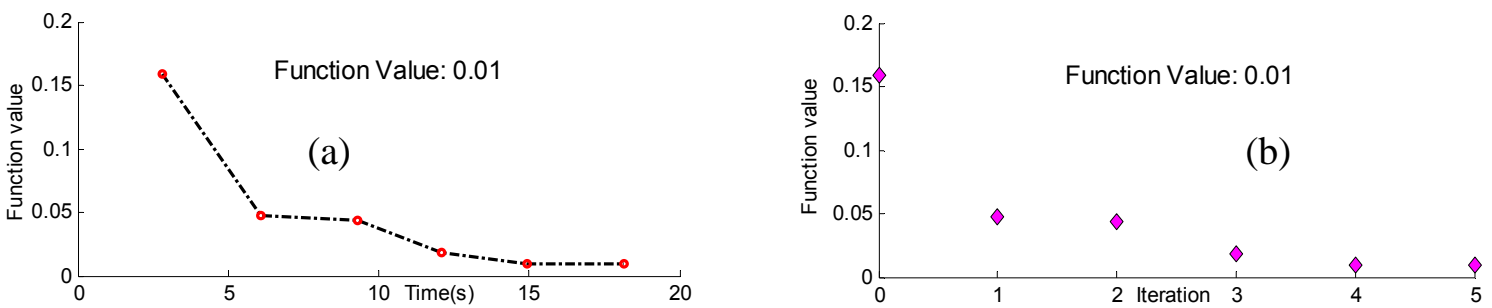

Figure 5 : Optimization by SQP, First section
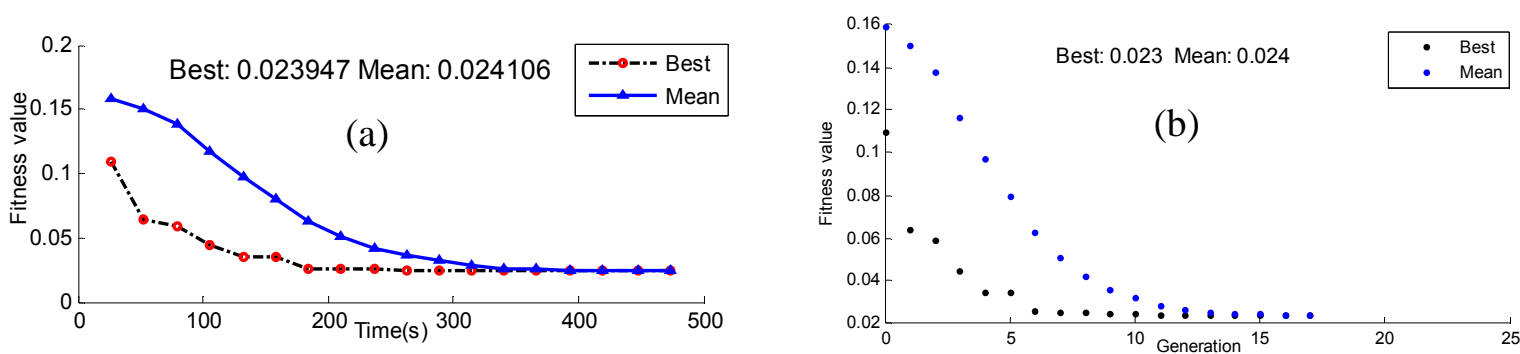

Figure 6 : Optimization by GA, First section
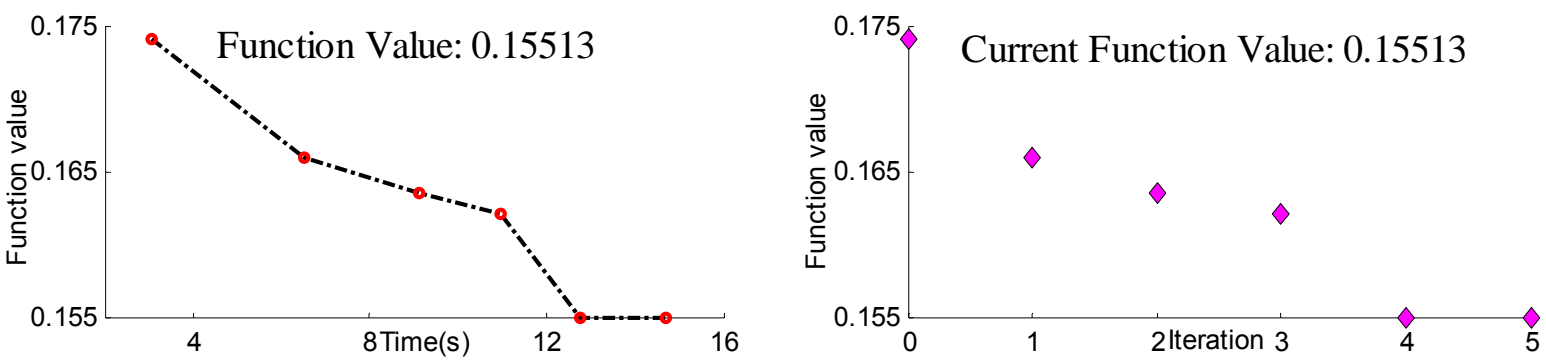

Figure 7 : Optimization by SQP, Second section
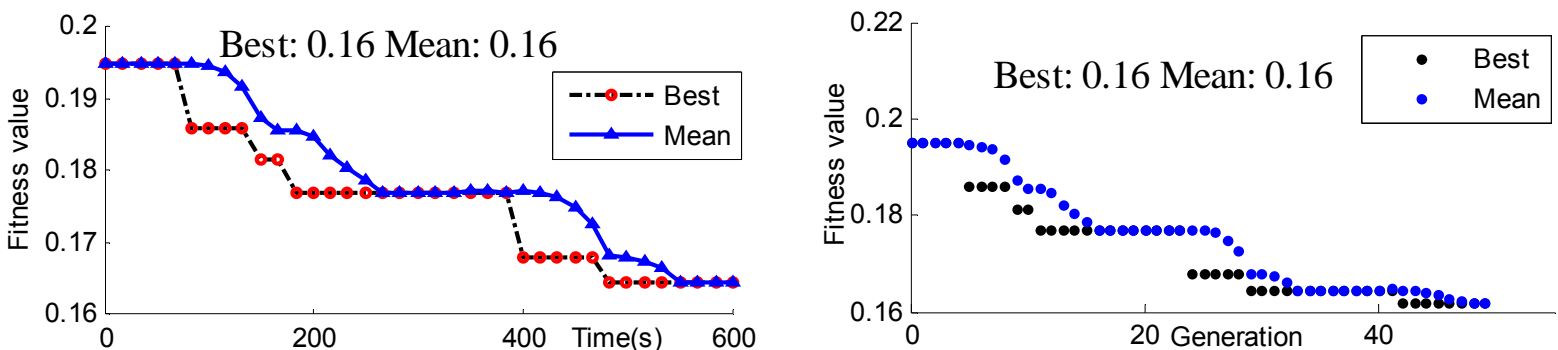

Figure 8 : Optimization by GA, Second section

\section{Discussion}

The GA is very sensitive to parameter settings such as crossover rate, mutation rate and population size (in this example, we used a population size of 200 , a crossover rate of 0.8 and a Gaussian mutation rate). If these parameters are correctly adjusted, enough population is created and random phenomena are used, the point obtained is almost sure to be a global minimum.

SQP does not always converge towards a global minimum. The end result depends highly on the initial guess point. If the initial point is correctly defined, the SQP is able to reach the minimum quickly.

In the case of the "double inclined plane", the results obtained confirm that the process plan is valid in terms of satisfying the functional tolerance. There is a small difference between the results obtained from these two different methods. So, in both approaches, there is no proof that the point obtained is the global minimum. The time required for the GA to find this point is nearly 30 times greater than that required using SQP.
The combined approach converges to the global minimum if all the defects parameters (DM and $\mathrm{DH}$ ) and the links (LHP and LGP) are assumed as independent variables.

As it could be found from Table 1, the obtained value from combined approach and optimization techniques are the same in the case of such quality constraints. When using the quality constraints type "Using the Measurement Results (independent parameters)" it is more meaningful to use combined approach because it is more rapid compared with optimization techniques (in the case of current example the elapsed time is less than 1 seconds). It can fast give to the process planner an initial idea about the quality of manufactured parts with the chosen process plan.

\begin{tabular}{|c|c|c|c|}
\hline \multirow{2}{*}{ Results } & \multicolumn{2}{|c|}{ Optimization } & \multirow{2}{*}{$\begin{array}{c}\text { Combined } \\
\text { Approach }\end{array}$} \\
\cline { 2 - 3 } & SQP & GA & C. \\
\hline GapGP $_{\min }(\mathrm{mm})$ & 0.01 & 0.024 & {$\left[\begin{array}{ll}0.01 & 0.30\end{array}\right]$} \\
\hline
\end{tabular}

Table 1 : Results for first section 


\begin{tabular}{|c|c|c|c|c|c|c|c|c|}
\hline \multicolumn{9}{|c|}{ Setup2 } \\
\hline \multicolumn{6}{|c|}{ Fixture } & \multicolumn{3}{|c|}{ Part (MWP) } \\
\hline \multicolumn{2}{|c|}{ Surface } & \multicolumn{3}{|c|}{ Surface deviation (DH) } & Constraints $(\mathrm{CH})$ & Surface & Surface deviation (DM) & Constraints $(\mathrm{CM})$ \\
\hline \multicolumn{2}{|c|}{ Plane 1S2 } & \multicolumn{3}{|c|}{$\mathrm{rx}_{1 \mathrm{~s} 2}, \mathrm{ry}_{1 \mathrm{~s} 2}, \mathrm{tz}_{1 \mathrm{~s} 2}$} & & Plane 1 & $\mathrm{rx}_{1}, \mathrm{ry}_{1}, \mathrm{tz}_{1}$ & \\
\hline \multicolumn{2}{|c|}{ Plane 2S2 } & \multicolumn{3}{|c|}{$\mathrm{rx}_{2 \mathrm{~s} 2}, \mathrm{ry}_{2 \mathrm{~s} 2}, \mathrm{tz}_{2 \mathrm{~s} 2}$} & & Plane 2 & $\mathrm{rx}_{2}, \mathrm{ry}_{2}, \mathrm{tz}_{2}$ & \\
\hline \multicolumn{2}{|c|}{ Plane 3S2 } & \multicolumn{3}{|c|}{$\mathrm{rx}_{3 \mathrm{~s} 2}, \mathrm{ry}_{3 \mathrm{~s} 2}, \mathrm{tz}_{3 \mathrm{~s} 2}$} & & Plane 3 & $\mathrm{rx}_{3}, \mathrm{ry}_{3}, \mathrm{tz}_{3}$ & \\
\hline \multicolumn{9}{|c|}{ Part/Fixture assembly } \\
\hline \multirow[b]{2}{*}{$\begin{array}{l}\frac{\overrightarrow{0}}{0} \\
\frac{0}{\frac{N}{0}} \\
\frac{.0}{1}\end{array}$} & \multirow[b]{2}{*}{ 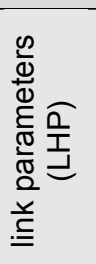 } & \multirow[b]{2}{*}{ 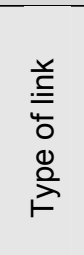 } & \multicolumn{6}{|c|}{ Positioning algorithm (CHP) } \\
\hline & & & 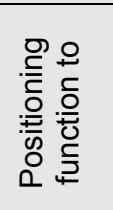 & \multicolumn{5}{|c|}{ Non-penetration condition } \\
\hline $\begin{array}{l}\frac{7}{d} \\
\stackrel{\frac{1}{L}}{\frac{1}{2}} \\
2\end{array}$ & $\begin{array}{l}\text { Irx } 1 \mathrm{~s} 2 \\
\text { Iry } \\
\text { Itz }_{1 s 2}\end{array}$ & 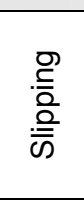 & $-\mid t z_{1 S 2}$ & \multicolumn{5}{|c|}{$\begin{array}{l}-25 \operatorname{Irx}_{1 \mathrm{~s} 2}+25 \operatorname{Iry}_{1 \mathrm{~s} 2}+\operatorname{Itz}_{1 \mathrm{~s} 2} \geq 0 \\
-25 \operatorname{Irx}_{1 \mathrm{~s} 2}-25 \operatorname{Iry}_{1 \mathrm{~s} 2}+\operatorname{Itz}_{1 \mathrm{~s} 2} \geq 0 \\
25 \operatorname{Irx}_{1 \mathrm{~s} 2}-25 \operatorname{Iry}_{1 \mathrm{ss}}+\operatorname{Itz}_{1 \mathrm{~s} 2} \geq 0 \\
25 \operatorname{Irx}_{1 \mathrm{~s} 2}+25 \operatorname{Iry}_{1 \mathrm{~s} 2}+\operatorname{Itz}_{1 \mathrm{~s} 2} \geq 0\end{array}$} \\
\hline $\begin{array}{l}\frac{7}{0} \\
\frac{0}{0} \\
\frac{1}{0} \\
0 \\
\infty\end{array}$ & $\begin{array}{l}\text { Iry } \\
\mathrm{Itz}_{2 \mathrm{~S} 2}\end{array}$ & $\begin{array}{l}\text { 응 } \\
\frac{.}{0} \\
\frac{.0}{5}\end{array}$ & $-\mid t z_{2 S 2}$ & \multicolumn{5}{|c|}{ 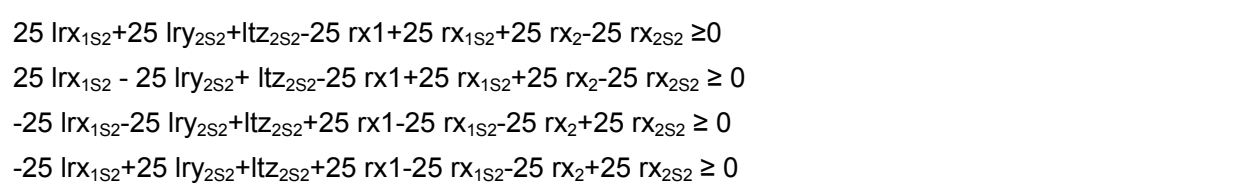 } \\
\hline 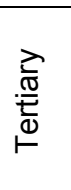 & $\mathrm{Itz} \mathrm{z}_{3 \mathrm{~S} 2}$ & 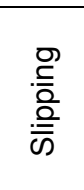 & $-\mid t z_{3 S 2}$ & \multicolumn{5}{|c|}{ 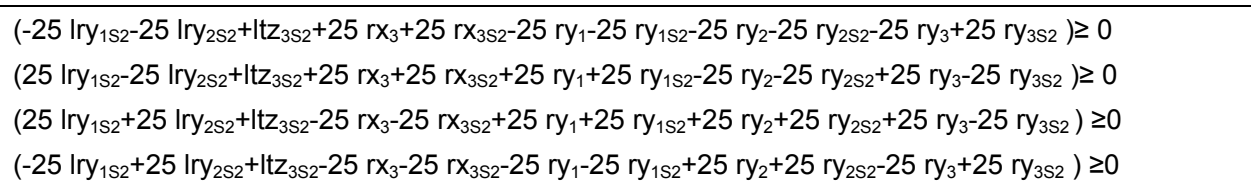 } \\
\hline
\end{tabular}

Table 2: Part/Fixture assembly procedure

\begin{tabular}{|c|c|c|c|}
\hline \multicolumn{2}{|c|}{$\begin{array}{l}\text { Machined } \\
\text { surface }\end{array}$} & $\begin{array}{l}\text { Verification } \\
\text { points }\end{array}$ & Results (mm) \\
\hline \multirow{3}{*}{ 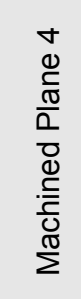 } & 1 & $(50,50,0)$ & $\begin{array}{l}\operatorname{GapGP}_{1}=\left[\begin{array}{ll}0.11 & 0.38\end{array}\right] \\
\text { GapGP }_{2}=\left[\begin{array}{ll}0.02 & 0.29\end{array}\right]\end{array}$ \\
\hline & 2 & $(0,50,50)$ & $\begin{array}{l}\operatorname{GapGP}_{3}=\left[\begin{array}{ll}0.03 & 0.28\end{array}\right] \\
\text { GapGP }_{4}=\left[\begin{array}{ll}0.12 & 0.37\end{array}\right]\end{array}$ \\
\hline & 3 & $(50,0,50)$ & $\begin{array}{l}\text { GapGP }_{5}=\left[\begin{array}{ll}0.01 & 0.30\end{array}\right] \\
\text { GapGP }_{6}=\left[\begin{array}{ll}0.10 & 0.39\end{array}\right]\end{array}$ \\
\hline
\end{tabular}

Table 3: Results obtained by combined approach

\subsection{Second section}

In the first section the combined approach and optimization techniques are compared when dealing with independent quality constraints. In this section we will explain how we can treat the dependent quality constraints. In this section the same process plan will be treated but the variation range of $\mathrm{DM}$ and $\mathrm{DH}$ parameters are defined by the variation zones as indicated in Table 4 .

The defects parameters variations, as mentioned in chapter 3.1, must be bound by the limits of the 3-D variation zones they represent. The MATLAB software was used for programming with a Pentium ${ }^{\circledR}, 3.2 \mathrm{GHz}$.

Both optimization algorithms (GA and SQP) were applied to minimize Sub I. Figure 8 represents the results obtained using the GA and Figure 7 represents those obtained using SQP.
For $\mathrm{MC}$, one million parts are produced virtually according to the defects generation described in Table 4 . The next step is to check the proportion of them that meet the functional tolerance. The GapGP are virtually measured between the part and the tolerance zone. The GapGP $P_{\text {min }}$ is then selected and will be retained as a result of the control. The distribution of the $\mathrm{GapGP}_{\min }$ values obtained for the 100000 parts is represented by the histogram Figure 9.

\section{Discussion}

The first point is that, the values obtained in section 2 by means of optimization techniques are more than those obtained in section 1. This is because of the fact that in section 1 all the defects parameters of a plane could have their extreme value simultaneously but in the case of section 2 there are limited by the bounds of 3D variation zone and they can not have their extreme value simultaneously. Actually the 3D bounded variation zone is closer to tolerance standard. The value obtained by MC, reflects accurately the real production. From Figure 9 it found that the worst value obtained for $G_{a p G P}$ in a little far from those obtained by optimization techniques. This raised from the fact that in real production the worst part might never been produced.

When using the optimization technique, the worst part is searched but with MC simulation the defects are generated randomly so the probability to find the worst part is very low.

The difference between the MC result and those of optimization (See Table 6) depends on the number of GapGP equation parameters. In fact this difference increases with the number of parameters. 


\begin{tabular}{|c|c|}
\hline \multicolumn{2}{|l|}{ Setup 1} \\
\hline \multicolumn{2}{|r|}{ Machining } \\
\hline Plane 1 & $\begin{array}{c}\mathrm{rx}_{1}, \mathrm{ry}_{1}, \mathrm{tz}_{1} \text { Planar variation zone } 0.01 \\
(\mathrm{~mm})\end{array}$ \\
\hline Plane 2 & $\begin{array}{c}\mathrm{rx}_{2}, \mathrm{ry}_{2}, \mathrm{tz}_{2} \text { Planar variation zone } 0.01 \\
(\mathrm{~mm})\end{array}$ \\
\hline Plane 3 & $\begin{array}{c}\mathrm{rx}_{3}, \mathrm{ry}_{3}, \mathrm{tz}_{3} \text { Planar variation zone } 0.01 \\
(\mathrm{~mm})\end{array}$ \\
\hline \multicolumn{2}{|l|}{ Set up 2} \\
\hline \multicolumn{2}{|r|}{ Positioning } \\
\hline $\begin{array}{c}\text { Plane } \\
1 S 2\end{array}$ & $\begin{array}{c}\mathrm{rx}_{1 \mathrm{~s} 2}, \mathrm{ry}_{1 \mathrm{~s} 2}, \mathrm{tz}_{1 \mathrm{~s} 2} \text { Planar variation zone } \\
0.02(\mathrm{~mm})\end{array}$ \\
\hline $\begin{array}{l}\text { Plane } \\
2 S 2\end{array}$ & $\begin{array}{c}\mathrm{rx}_{2 \mathrm{~s} 2}, \mathrm{ry}_{2 \mathrm{~s} 2}, \mathrm{tz}_{2 \mathrm{~s} 2} \text { Planar variation zone } \\
0.02(\mathrm{~mm})\end{array}$ \\
\hline $\begin{array}{l}\text { Plane } \\
3 \text { S2 }\end{array}$ & $\begin{array}{c}r x_{3 s_{2}}, r_{3 s_{2}}, t_{z} z_{22} \text { Planar variation zone } \\
0.02(\mathrm{~mm})\end{array}$ \\
\hline \multicolumn{2}{|r|}{ Machining } \\
\hline Plane 4 & $\begin{array}{c}\text { rx4, ry4, tz4 Planar variation zone } \\
0.006(\mathrm{~mm})\end{array}$ \\
\hline
\end{tabular}

Table 4: Deviation range for "Double inclined plane" manufacturing process

\begin{tabular}{|c|c|c|c|}
\hline \multirow{2}{*}{ Results } & \multicolumn{2}{|c|}{ Optimization } & \multirow{2}{*}{$\begin{array}{c}\text { MC } \\
\text { simulation }\end{array}$} \\
\cline { 2 - 3 } & SQP & GA & \\
\hline GapGP $_{\min }(\mathrm{mm})$ & 0.15 & 0.16 & 0.17 \\
\hline
\end{tabular}

Table 6: Results for second section

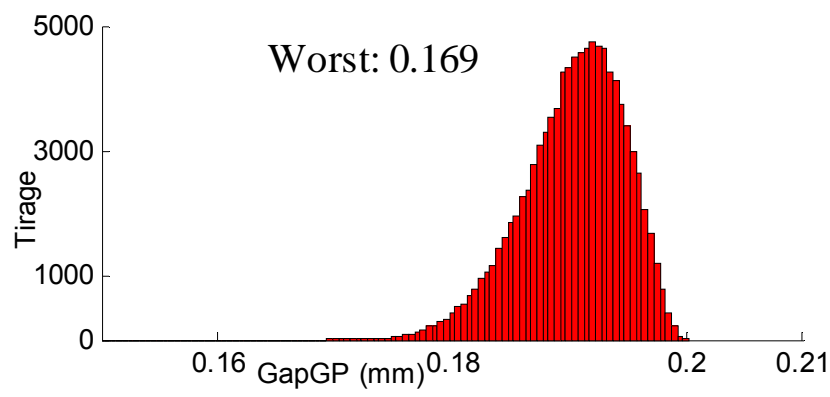

Figure 9: $\mathrm{MC}$ results

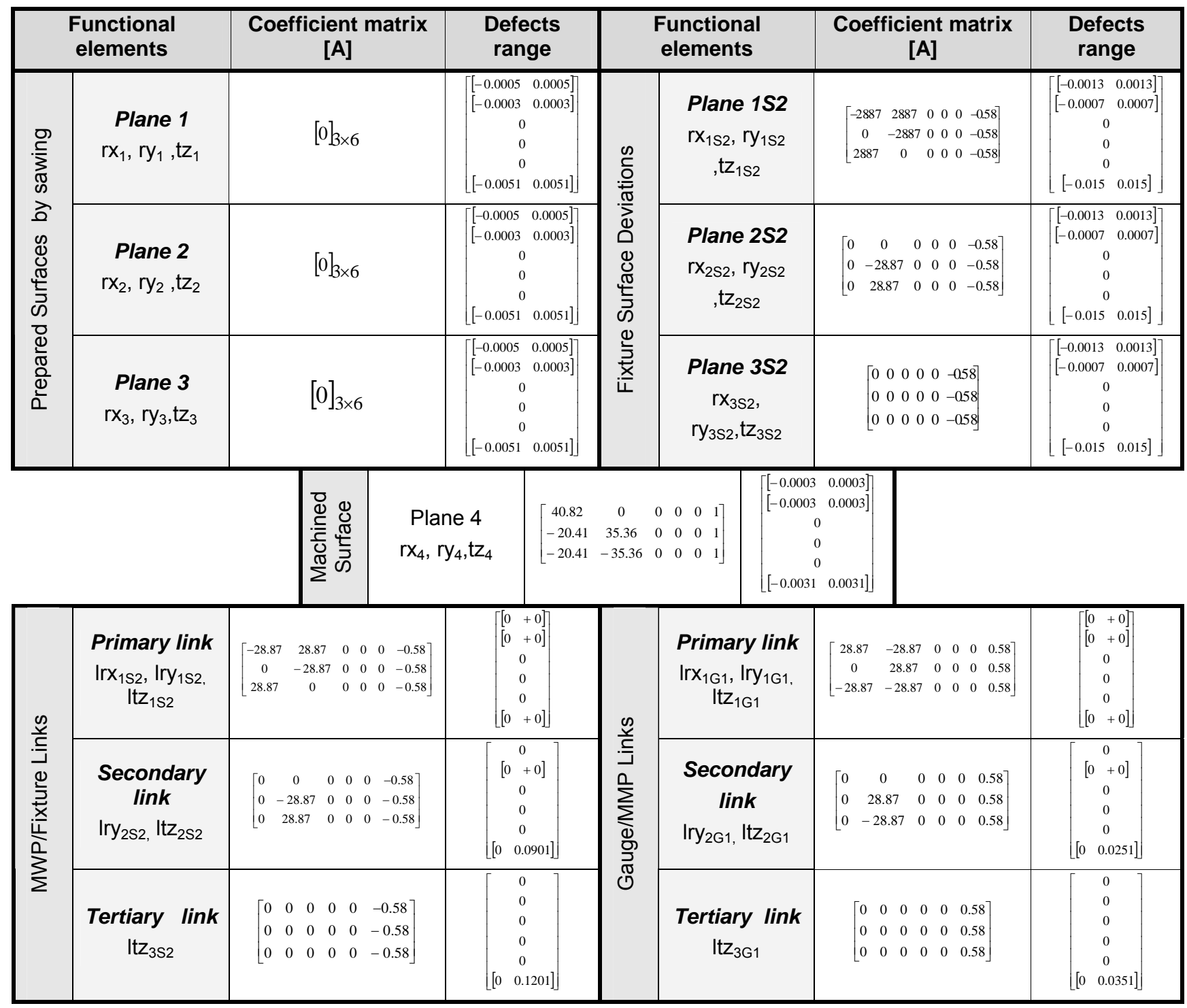

Table 5 : Functional elements (FE) and their related coefficient matrices and Torsors with intervals 


\section{CONCLUSION}

This paper presented three different solution techniques for searching the worst part within a process plan from geometrical point of view for the aims of tolerance analysis in multi stage machining operations.

These techniques are compared through a 3D example and their characteristics are discussed.

Actually the optimization techniques and MC can deal with all types of quality constraints. The combined approach can deal with quality constraints with independent parameters.

From the elapsed time point of view, combined approach is very fast. The optimization techniques need much more time and based on the complexity of the problem the time could become long. The MC need much more time compared with optimization techniques. The defects are generated randomly therefore for well covering the variation scope; the number of virtual produced part should be big enough (more than 100000 parts, see[14]) . This leads to long calculation time (30 minutes in the case of current example).

From convergence point of view, when dealing with quality constraints with independent parameters, it is possible to check the results obtained by optimization techniques with those of combined approach to make sure that the obtained point is the global minimum.

When dealing with quality constraints with dependent parameters, in both optimization approaches (GA and $S Q P)$, there is no proof that the point obtained is the global minimum. The time required for the GA to find the optimum point is much more than that required using SQP. However, this time advantage for SQP is offset by the time needed to locate a suitable initial point.

As it is under lined before, the value obtained by $M C$ is a little far from optimization, this could be over stepped by using more efficient random generator for well covering the variation scope.

\section{REFERENCES}

[1] F. Villeneuve, F. Vignat, 2005, Manufacturing process simulation for tolerance analysis and synthesis,In: Bramley, A., Brissaud, D., et al., Eds, Advances in Integrated Design and Manufacturing in Mechanical Engineering, presented at: 5th International Conference on Integrated Design and Manufacturing in Mechanical Engineering, Bath, ENGLAND. pp. 189-200

[2] M. Kamali nejad, F. Vignat, F. Villeneuve, 2008, Tolerance Analysis in Multi-Operational Machining Process Based on the Model of Manufactured Part In: 5th International Conference on Digital Enterprise Technology, Nantes, France

[3] W. Ghie, L. Laperriere, A. Desrochers, 2003, A unified Jacobian-torsor model for analysis in computer aided tolerancing,In: Gogu, G., Coutellier, D., et al., Eds, Recent Advances in Integrated Design and Manufacturing in Mechanical Engineering, presented at: 4th International Conference on Integrated Design and
Manufacturing in Mechanical Engineering, Clermont Fernand, FRANCE. pp. 63-72

[4] M. Kamali nejad, A. Desrochers, F. Vignat, et al., 2008, Tolerance Analysis in Machining, An Approach Combining The Model of Manufactured Part and The Jacobian-torsor Model, presented at: ASME 28th Computers and Information in Engineering Conference ( $\mathrm{CIE}$ ), New York City, US

[5] F. Vignat, F. Villeneuve, M. Kamalinejad, 2008, From the nominal model to a Model of Manufactured Parts in a CAD context, presented at: CIRP Design Conference Netherlands.

[6] K. Whybrew, G. Britton, D. Robinson, et al., 1990, A graph-theoretic approach to tolerance charting, The International Journal of Advanced Manufacturing Technology, 5:175-183

[7] Q. Huang, J. J. Shi, 2003, Part dimensional error and its propagation modeling in multi-operational machining processes, Journal of Manufacturing Science and Engineering-Transactions of the Asme, 125:255-262

[8] S. Y. Zhou, Q. Huang, J. J. Shi, 2003, State space modeling of dimensional variation propagation in multistage machining process using differential motion vectors, leee Transactions on Robotics and Automation, 19:296-309

[9] J. P. Loose, S. Y. Zhou, D. Ceglarek, 2007, Kinematic analysis of dimensional variation propagation for multistage machining processes with general fixture layouts, leee Transactions on Automation Science and Engineering, 4:141-152

[10] S. H. Huang, Q. Liu, R. Musa, 2004, Tolerancebased process plan evaluation using Monte Carlo simulation, International Journal of Production Research, 42:4871-4891

[11] F. Vignat, F. Villeneuve, 2007, Simulation of the Manufacturing Process, Generation of a Model of the Manufactured Parts,In: Cunha, P., Maropoulos, P., Eds, Digital Enterprise Technology: Perspectives and Future Challenges presented at: 3rd International Conference on Digital Enterprise Technology, Bath. pp. 545-552

[12] F. Villeneuve, F. Vignat, 2007, Simulation of the manufacturing process in a tolerancing point of view: Generic resolution of the positioning problem,In: Davidson, J. K., Ed., Models for Computer Aided Tolerancing in Design and Manufacturing presented at: Models for Computer Aided Tolerancing in Design and Manufacturing, Arizona. pp. 179-189

[13] S. Tichadou, M. Kamali nejad, F. Vignat, et al., 2007, 3-D manufacturing dispersions: two experimental applications. In: 10th CIRP International Conference on Computer Aided Tolerancing, Erlangen, Germany

[14] F. Germain, PhD Thesis, 2007, "Statistical 3D Tolerancing ", SAVOIE University 